\title{
Study of the variation of B with $\mathrm{Sr}$
}

\author{
Mathilde Morvan ${ }^{1 \mathrm{a}}$, Mathilde Vernay ${ }^{1}$ and Pierre Breul ${ }^{1}$ \\ ${ }^{1}$ Institut Pascal, Polytech Clermont-Ferrand, Université Blaise Pascal, Clermont-Ferrand, France
}

\begin{abstract}
Skempton coefficient B is commonly used to verify the saturation of a sample before triaxial testing. This coefficient is obtained during undrained isotropic consolidation and is defined as the ratio between the increment of pore pressure u measured and the imposed increment of isotropic stress. This coefficient varies between 0 for dry soils and 1 for saturated soils. Many studies on liquefaction of unsaturated soils were published using Skempton coefficient B to represent saturation degree $\mathrm{Sr}$ of soil. On the first hand, this variation of B coefficient with saturation degree is mostly due to the compressibility of air in the pores. On the second hand, we also know that the presence of air as a fluid phase gives birth to suction after equilibrium is reached inside the sample. The higher the suction, the stiffer the soil skeleton. These two phenomena are opposite. Their effects in laboratory testing depend on the experimental apparatus. For example, if we consider an unsaturated triaxial device, we will have to take suction into account. On the contrary if we plan to break the menisci just before measuring B, suction equilibration will not occur. Experimental tests were performed to show the difference between these two cases and to study the equilibrium phase. Based on these observations, this article presents new relationships that permit to calculate saturation degree with a given Skempton coefficient with different hypotheses and with different experimental devices. These results are confronted to the commonly used relation given by Lade and the difference between all these calculations is studied.
\end{abstract}

\section{Introduction}

Nowadays, Skempton coefficients $A$ and $B$ [1] are commonly used in earth dam study. They were proposed by Skempton (1954) in order to anticipate pore pressure changes due to mechanical stresses:

$$
\Delta u=B\left[\Delta \sigma_{3}+A\left(\Delta \sigma_{1}-\Delta \sigma_{3}\right)\right]
$$

Practical use of these parameters was exposed by Bishop [2].

Coefficient B is linked to saturation degree so it can also be used to verify saturation of a sample during triaxial testing. Coefficient B can be defined as the ratio between increment of pore water pressure and increment of isotropic pressure when a sample is subjected to an increment of isotropic pressure. So we have:

$$
B=\frac{d u_{w}}{d p}
$$

For a saturation degree of $100 \%$, we get $B=1$ if we consider water and skeleton grain as incompressible.

Some authors try to give a unique relation between B and Sr. The usual one was determined by Skempton [1]. This equation is widely used during undrained triaxial tests and liquefaction tests for example $[5,6,7,8]$.

$$
B=\frac{d u_{w}}{d p}=\frac{1}{1+\frac{n C_{v}}{C_{c}}}
$$

Where $C_{c}$ is the compressibility of skeleton, $C_{v}$ fluid compressibility and $n$ sample porosity.

During their study of membrane penetration, Lade and Hernandez [3] used the equation giving $B$ in function of Sr using the results of Hilf [4] and Skempton [1].

Schuurman [9] modified this equation taking equilibrium of air bubbles into account. To perform these calculations, he assumed that the number of bubbles is constant during the test. This hypothesis was not verified [10].

More recently, some researchers give new equations to obtain this relationship. Hasan and Fredlund [11] developed a method to obtain $B_{w}$ and $B_{\text {a }}$ depending on the studied fluid (water or air) for unsaturated state. They considered two compressibility parameters for skeleton and air [12]. These parameters and the obtained relationship do not offer an easy calculation.

Boutonnier [13] developed a methodology to obtain B for different saturation degree ranges.

In this article we propose to study this relationship between $\mathrm{B}$ and $\mathrm{Sr}$ and parameters related firstly using a classical approach, then an approach considering an equilibrium before the application of the isotropic pressure increment and finally to consider the elastoplastic behavior of the soil skeleton.

$\overline{{ }^{a} \text { Corresponding author: mathilde.morvan@ } @ u n i v-b p c l e r m o n t . f r}$ 


\section{Usual simulation}

In this first part, we used Skempton and Lade [1, 3] hypotheses so we can obtain a simple relationship between $\mathrm{B}$ and $\mathrm{Sr}$. We made the assumption that effective stress of saturated soils is still valid and that stress variation is small so we have an elastic behavior of soil. We also consider that no phase change occurs.

To measure B coefficient, triaxial cell is used. So in this simulation and the following ones, we considered a revolution symmetry and used following parameters:

$$
\begin{aligned}
& \quad p=\frac{1}{3}\left(\sigma_{1}+2 \sigma_{3}\right) ; q=\sigma_{1}-\sigma_{3} ; \\
& \varepsilon_{p}=\varepsilon_{1}+2 \varepsilon_{3} ; \varepsilon_{q}=\frac{2}{3}\left(\varepsilon_{1}-\varepsilon 3\right)
\end{aligned}
$$

Where subscript 1 is linked to vertical direction and subscript 3 is linked to radial direction.

Mechanical behaviour can be described by:

$$
d \varepsilon_{p}=-\frac{d V_{a}}{V_{0}}=\frac{\kappa}{p^{\prime}\left(1+e_{0}\right)} d p^{\prime}
$$

With $p^{\prime}=p-u_{w}, u_{a}=u_{w}$ and $\kappa$ represents the slope of elastic loading lines in a $(e-\log \mathrm{p})$ plane.

We also considered that water (subscript $\mathrm{w}$ ) and grains (subscript s) are uncompressible: $d V_{w}=d V_{s}=0$

Air pore pressure is calculated using Boyle's law:

$$
P_{a} V_{a}=P_{a 0} V_{a 0}=\left(P_{a 0}+d P_{a}\right)\left(V_{a 0}+d V_{a}\right)
$$

With $P_{a}$ absolute air pore pressure and $V_{a}$ air volume.

So we get

$$
\left(P_{a 0} d V_{a}\right)+\left(V_{a 0} d P_{a}\right)=0
$$

Considering that $d V_{a} d P_{a}$ is negligible,

Knowing that:

$$
V_{a}=\frac{e_{0}}{1+e_{0}} V_{0}\left(1-S_{r}\right)
$$

We can obtain:

$$
\begin{gathered}
B=\frac{d u_{w}}{d p}=\frac{d u_{a}}{d p} \\
B=\frac{\kappa}{\kappa+\frac{p_{0}{ }^{\prime} V_{a 0}\left(1+e_{0}\right)}{V_{0} P_{a 0}}}=\frac{1}{1+\frac{p_{0}{ }^{\prime} e_{0}\left(1-S_{r}\right)}{\kappa P_{a 0}}}
\end{gathered}
$$

Parameters used for this first simulation are summarized in Table1.

Table 1. Parameters used in usual simulation.

\begin{tabular}{|c|l|}
\hline Parameter & Value \\
\hline$\kappa$ & 0.006 \\
\hline$p_{0}$ & $100 \mathrm{kPa}$ \\
\hline$u_{a 0}=u_{w 0}$ & $30 \mathrm{kPa}$ \\
\hline$e_{0}$ & 0.8 \\
\hline$p_{a 0}$ & $130 \mathrm{kPa}$ \\
\hline
\end{tabular}

These hypotheses give the relationship shown in Fig.1. We can see that the relationship between $\mathrm{B}$ and $\mathrm{Sr}$ is monotonic and that for $\mathrm{B}>0.2, \mathrm{Sr}>95 \%$. So even if $\mathrm{B}$ is low, saturation degree is still high. So B is a good mean to evaluate near saturation value of saturation degree.

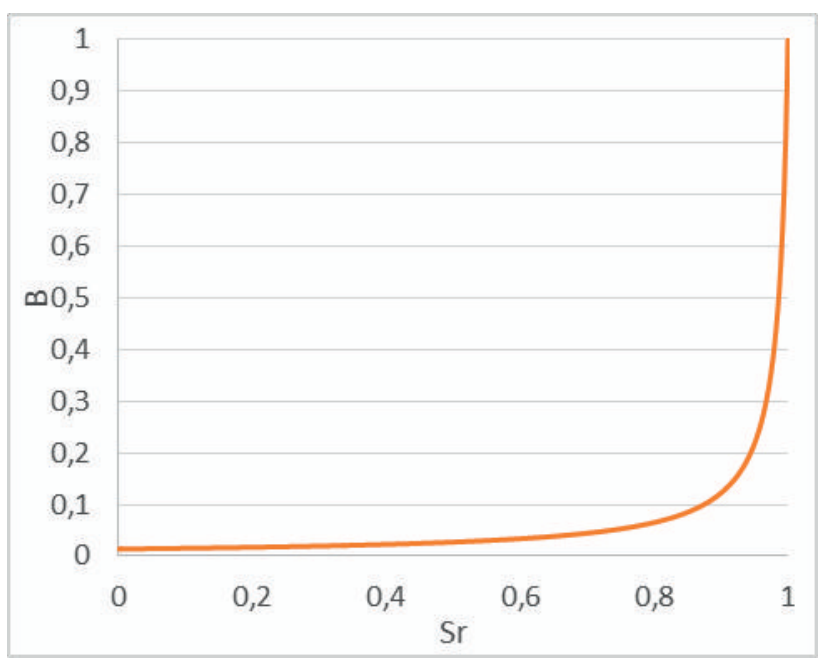

Figure 1. Evaluation of B using simple method

Two parameters seems to influence this relationship: isotropic elastic modulus and initial void ratio. So we propose to study their influence.

Fig. 2 presents variation of $\mathrm{B}, \mathrm{Sr}$ relationship with kappa parameter. As we could forecast, this parameter has a significant effect on the curves. The more rigid is the soil, the lower B is for a same saturation degree. For example, Skempton coefficient at a saturation degree of $90 \%$, for $\kappa=0.06$ we get $\mathrm{B}=0.58$ and for $\kappa=0.0006, \mathrm{~B}=0.01$. This huge difference show that the determination of this parameter is essential to determine B.



Figure 2. Effect of kappa on B simulations

If we consider the relationship variations with $\mathrm{e}_{0}$ (Fig.3) we can see that for a void ratio from 0.6 to 1.2 observed difference is low. Skempton coefficients for a saturation degree of $90 \%$ for $\mathrm{e}_{0}=0.6, \mathrm{~B}=0.15$ and $\mathrm{e}_{0}=1.2, \mathrm{~B}=0.08$. 


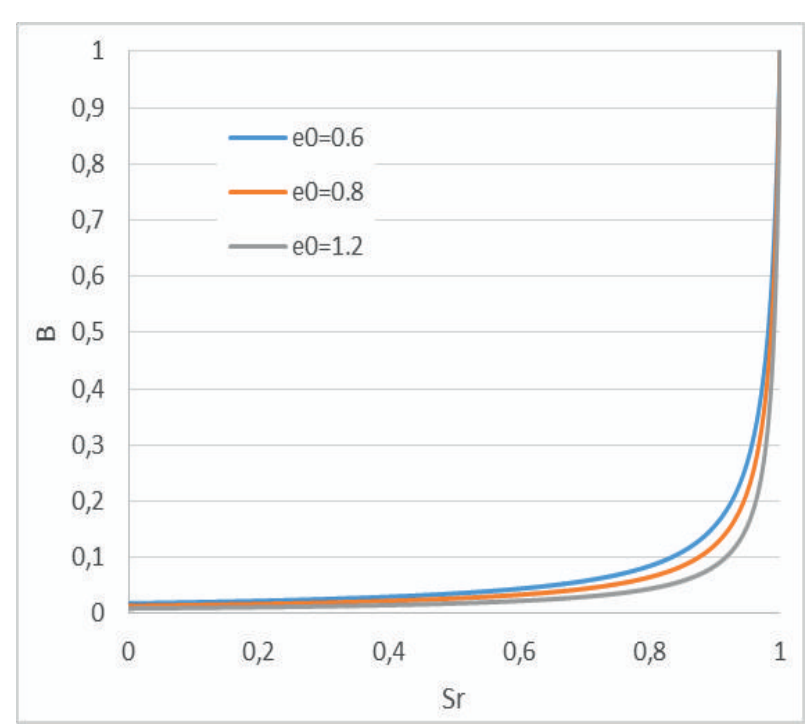

Figure 3. Effect of initial void ratio on B simulations

\section{Simulation with initial suction equilibrium}

When soil is unsaturated, voids are filled with two fluids. At equilibrium it causes a suction responsible for changes of mechanical behavior of soil skeleton. Suction is given as the difference between air pore pressure and water pore pressure:

$$
s=u_{a}-u_{w}
$$

If we measure $B$ after waiting the equilibrium, calculation have to take the effects of suction into account. So we have an increase of sample rigidity with saturation degree decrease. In this simulation, parameters will depend on mechanical behavior of soil, but also on hydromechanical behaviour. Two main parameters can be taken into account, these two parameters govern water retention curve and vary with kind of soil.

Table 2. Parameters used in elastic simulation

\begin{tabular}{|c|c|}
\hline Parameter & value \\
\hline$\kappa$ & 0.006 \\
\hline$p_{0}$ & $100 \mathrm{kPa}$ \\
\hline$e_{0}$ & 0.8 \\
\hline$p_{a 0}$ & $130 \mathrm{kPa}$ \\
\hline$s_{e}$ & 20 \\
\hline$\alpha$ & 0.8 \\
\hline
\end{tabular}

An incremental calculation for each saturation degree is needed to obtain Skempton coefficient for this simulation.

For each saturation degree we can calculate suction thanks to Brooks and Corey law [14]. Parameters are $s_{e}$ and $\alpha . s_{\mathrm{e}}$ is air entry suction and depends on grain size and $\alpha$ depends on grain size distribution uniformity. These two parameters vary with the type of soil. These simulation were performed using a bijective relationship between suction $\mathrm{s}$ and saturation degree $\mathrm{Sr}$.
This obtained suction leads us to initial water pore pressure. We verified experimentally that when equilibrium is reached, water pore pressure is negative and air pore pressure remains equal to atmospheric pressure.

So we get:

$$
u_{w}=u_{a}-s
$$

Initial effective stress is calculated using enlarged effective stress concept with a simplified law [15].

$$
\sigma^{\prime}=\sigma-u_{a}+s S_{r}=\sigma-u_{a}+\chi
$$

For mechanical behavior, we choose a classical law with an isotropic modulus depending on effective mean stress as in previous simulation.

The main difference between this new simulation and the previous one is the effect of suction that causes effective stress variation whereas stress increment remains the same. We verified that $d p$ has no influence on the simulation results.

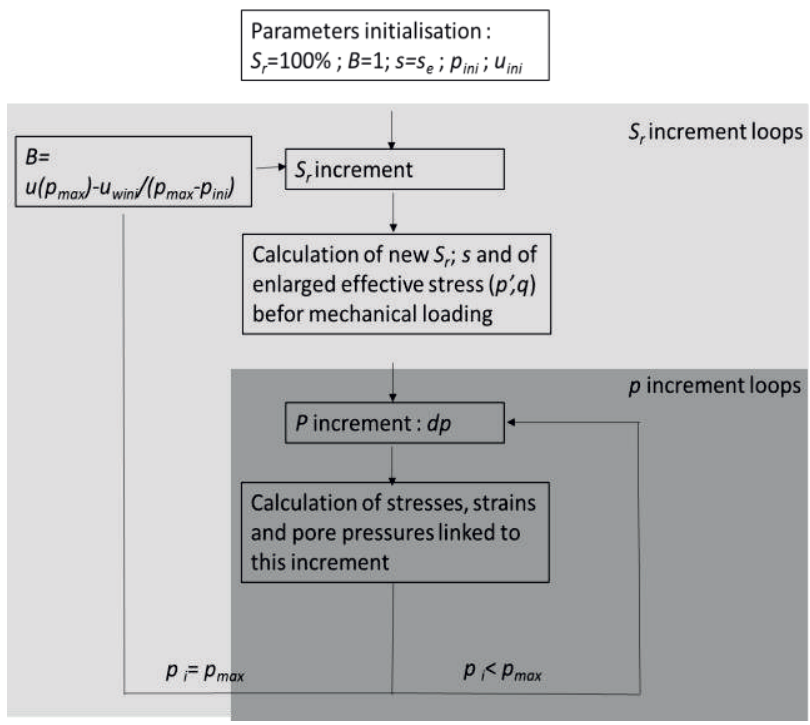

Figure 4. Diagram of simulation algorithms

Then to study the influence of parameters of the water retention curve, we made some simulations for different $\alpha$ and air entry suction $s_{e}$. For a fine grain size distribution, $\mathrm{s}_{\mathrm{e}}$ will be high and on the contrary for coarse grain size distribution it can be lower than $10 \mathrm{kPa}$.

Fig. 5 presents the results of simulations for four values of $\mathrm{s}_{\mathrm{e}}: 5 \mathrm{kPa}, 20 \mathrm{kPa}, 100 \mathrm{kPa}, 1000 \mathrm{kPa}$. We can see here that $\mathrm{s}_{\mathrm{e}}$ has also a significant effect on $B-S_{r}$ relationship. The higher $\mathrm{s}_{\mathrm{e}}$, the lower $B$ is. Previous relationship curve is close to the curve we can obtain for a sand which behaviour is little influenced by suction. So for a granular material, previous relationship giving $\mathrm{B}$ variation with $\mathrm{Sr}$ is accurate enough. For fine soils, this relationship can lead to a significant underestimation of saturation degree for a given $\mathrm{B}$. 


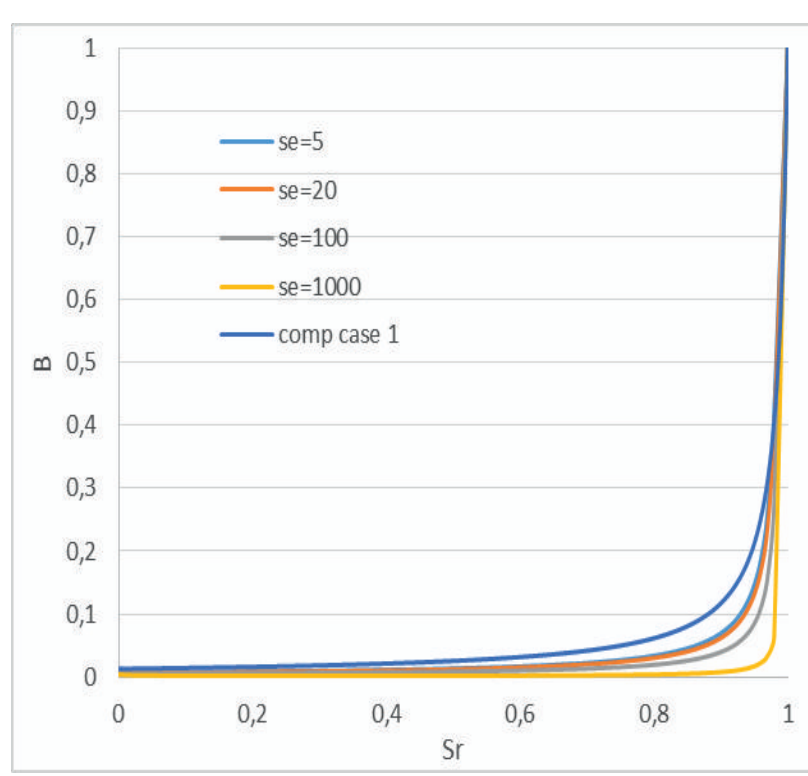

Figure 5. Dependency of B on air entry suction for second simulation

\section{Elastoplastic simulation}

We wanted to be more accurate in this study of B versus Sr relationship. So we propose a third simulation taking elasto-plasticity into account with enlarged effective stress. Initial state of sample will give birth to different consolidation state. A normally consolidated sample will quickly develop plastic strains and an overconsolidated sample would develop mainly elastic trains. Triaxial apparatus allows us to rebuild samples but also to work on undisturbed samples, so each case can occur.

We choose BDNS model [16] for this calculation. In this model, enlarged effective stress concept is used as well as the bounding surface theory that permits a smooth transition between elastic and plastic domains. It gives us a matrix $\boldsymbol{A}$ that linked the strain vector to the stress vector where suction is added. So it gives:

$$
\left(\begin{array}{l}
d \varepsilon_{p} \\
d \varepsilon_{q}
\end{array}\right)=\left(\begin{array}{lll}
A_{11} & A_{12} & A_{13} \\
A_{21} & A_{22} & A_{23}
\end{array}\right)\left(\begin{array}{c}
d p^{\prime} \\
d q \\
d s
\end{array}\right)
$$

As for the previous simulation, we need two incremental loops: the first one is based on an increment of saturation degree and the second one, for each saturation degree increment, an elastoplastic calculation is performed for a given isotropic pressure increment.

For this calculation, after isotropic pressure increment, we consider that isotropic pressure increment is imposed too fast to reach suction equilibrium in this loop. So we consider that $\chi$ remains constant. It could be interesting to study the influence of rate of load application (equilibrium can be reached or not).

To obtain the result of $d p$ isotropic pressure on pore pressure increment, we have to separate this matrix law. To achieve this point, some hypotheses are necessary. For this calculation, we make the assumption that suction remains constant so it gives $d u_{a}=d u_{w}$. Saturation degree increment is inserted in strain increment vector. Variation of air pore pressure is obtained using Boyle's law. It adds another dimension to the matrix with $d u_{a}$ as stress variable and $d V_{a}$ as strain variable.

$$
\left(\begin{array}{l}
d \varepsilon_{p} \\
d \varepsilon_{q} \\
d S_{r} \\
d V_{a}
\end{array}\right)=\left(\begin{array}{llll}
D_{11} & D_{12} & D_{13} & D_{14} \\
D_{21} & D_{22} & D_{23} & D_{24} \\
D_{31} & D_{32} & D_{33} & D_{34} \\
D_{41} & D_{42} & D_{43} & D_{44}
\end{array}\right)\left(\begin{array}{c}
d p \\
d q \\
d s \\
d u_{a}
\end{array}\right)
$$

So we obtained a matrix $\boldsymbol{D}$ with 16 unknown variables that links $\left(d \varepsilon_{p}, d \varepsilon_{q}, d S_{r}, d V_{a}\right)$ to $\left(d p^{\prime}, d q, d s, d u_{a}\right)$.

To study the influence of $d p$, first we have to separate mechanic and hydric variables. Using the enlarged effective stress definition, we get:

$$
d p^{\prime}=d p-d u_{a}+d \chi=d p-d u_{a}+s d S_{r}+S_{r} d s
$$

Relationship between $d s$ and $d S_{r}$ gives us:

$$
d S_{r}=-S_{r} \frac{\alpha}{s} d s=D_{33} d s
$$

So we have:

$$
d p^{\prime}=d p-d u_{a}+S_{r}(1-\alpha) d s
$$

Using Boyle's law incremental form:

$$
d V_{a}=-\frac{V_{a}}{P_{a}} d u_{a}=D_{44} d s
$$

Increment of volumetric strain is given by:

$$
d \varepsilon_{p}=D_{11} d p+D_{12} d q+D_{13} d s+D_{14} d u_{a}
$$

With $D_{11}=A_{11} ; D_{12}=A_{12}, D_{13}=A_{13}+A_{11}(1-\alpha) S_{r}$; $D_{14}=-A_{11}$

We made the assumption that $d q=d \chi=0$

So volumetric strain increment can be simplified:

$$
d \varepsilon_{p}=D_{11} d p+D_{14} d u_{a}
$$

We also have:

$$
d u_{a}=-\frac{P_{a}}{V_{a}} d V_{a}=\frac{P_{a}}{V_{a}} V_{0} d \varepsilon_{p}
$$

So we get:

$$
d \varepsilon_{p}=\frac{A_{11}}{1+A_{11} \frac{P_{a}}{V_{a}} V_{0}} d p
$$

With $d u_{a}=d u_{w}$ :

$$
d u_{a}=d u_{w}=\frac{P_{a}}{V_{a}} V_{0} \frac{A_{11}}{1+A_{11} \frac{P_{a}}{V_{a}} V_{0}} d p
$$

For this simulation, elastic parameters are the same as in previous simulations. Every parameters are given in table 3. Parameters $\Gamma, \rho$ and $M$ defined the shape of the bounding surface. $\lambda_{0}$ is the plastic isotropic modulus. $A_{\text {ini }}$ gives the initial position of the surface.

Simulations for a normally consolidated sample and for an overconsolidated sample are given Fig. 6. For these two simulations the difference is not located at high saturation degrees but also at low saturation degrees. For a saturation degree of $90 \%$, we obtained B of 0.4 for a normally consolidated sample and B equal to 0.04 for an overconsolidated sample. 
Table 3. Parameters used in elasto-plastic simulation

\begin{tabular}{|c|l|}
\hline Parameter & value \\
\hline$\kappa$ & 0.006 \\
\hline$p_{0}$ & $100 \mathrm{kPa}$ \\
\hline$e_{0}$ & 0.8 \\
\hline$p_{a 0}$ & $130 \mathrm{kPa}$ \\
\hline$s_{e}$ & 20 \\
\hline$\alpha$ & 0.8 \\
\hline$\lambda_{0}$ & 0.2 \\
\hline$\Gamma$ & 1.85 \\
\hline$\rho$ & 2 \\
\hline $\mathrm{M}$ & 1.6 \\
\hline $\mathrm{A}_{\mathrm{ini}}$ & 0 or $10 \mathrm{kPa}$ \\
\hline
\end{tabular}

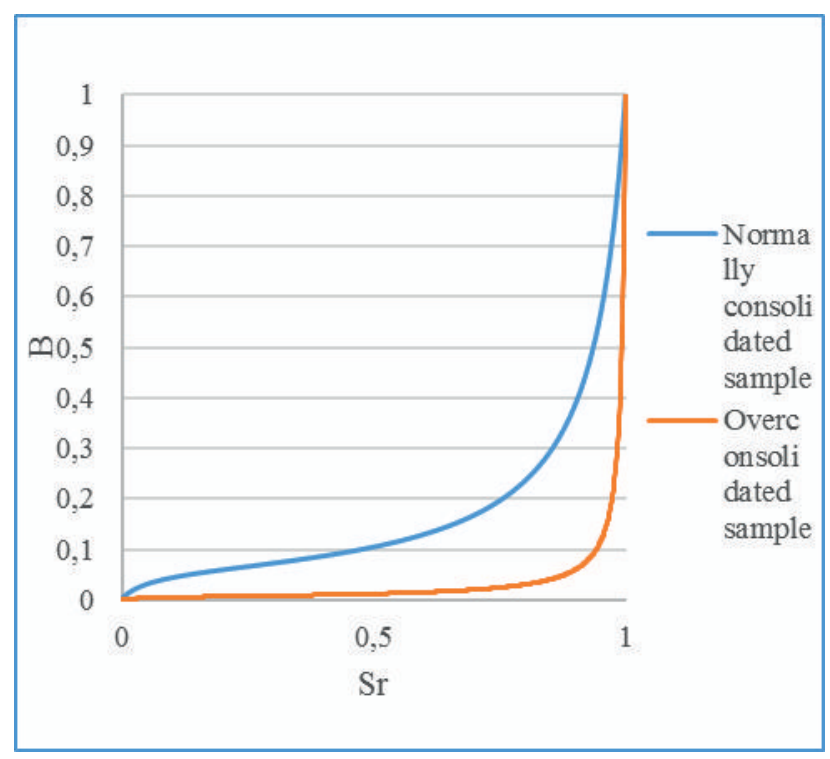

Figure 6. Dependency of B toward consolidation state

\section{Conclusion}

To conclude, this article proposed three simulations to obtain Skempton coefficient B knowing saturation degree Sr. We enlighten that the initial relationship depends principally on two parameters. The two other simulations show that two other parameter could be taken into account: water retention curve and consolidation state of studied soil. These two parameters need to be further studied and these results need to be compared with experimental data.

\section{References}

1. A. W. Skempton, The Pore-Pressure Coefficients A and B. Géotechnique, 4, 143-147 (1954).
2. A. W. Bishop, The Use of Pore-Pressure Coefficients in Practice. Géotechnique, 4, 148-152 (1954).

3. P. V. Lade, \& S. B. Hernandez, Membrane penetration effects in undrained tests. Journal of the geotechnical engineering division, 103, 109-125 (1977).

4. J.W. Hilf, Estimating construction pore pressure in rolled earth dams. Proceedings of 2 nd International Conference on Soil Mechanics and Foundation Engineering. Rotterdam, vol. 3, p.234-240 (1948)..

5. K. Mitsuji, Numerical simulations for development of liquefaction. Countermeasures by use of partially saturated sand. The 14th World Conference on Earthquakes Engineering. Beijing, China (2008).

6. Y. Yoshimi, K. Tanaka, K. Tokimatsu, Liquefaction resistance of a partially saturated sand. Soils and Foundations, 29, 157-162 (1989).

7. N. Della, Laboratory testing of the Monotonic behavior of partially saturated sandy soil. Earth Sciences Research Journal, 14, 181-186. (2010)

8. M. E. Raghunandan, \& A. Juneja, A study on the liquefaction resistance and dynamic properties of desaturated sand. Electronic Journal of Geotechnical Engineering, 16, 109-123 (2011).

9. I. E. Schuurman. The Compressibility of an Air/Water Mixture and a Theoretical Relation Between the Air and Water Pressures. Géotechnique, 16, 269-281 (1996).

10. A.Bendi-Ouis, N. Abou-Bekr, S. Taibi,, J. M. Fleureau,Prédiction du coefficient de pression interstitielle B: Etat de l'art. Colloque International Sols Non Saturés et Environnement UNSAT09. Tlemcen, Algérie (2009).

11. J. U. Hasan, D. G. Fredlund, Pore pressure parameters for unsaturated soils. Canadian Geotechnical Journal, 17, 395-404 (1980).

12. D. G. Fredlund, N. R. Morgenstern, Constitutive relations for volume change in unsaturated soils. Canadian Geotechnical Journal, 13, 261-276 (1976).

13. L. Boutonnier, Comportement hydromécanique des sols fins proches de la saturation. Cas des ouvrages en terre : coefficient $\mathrm{B}$, déformations instantanées et différées, retrait/gonflement. $\mathrm{PhD}$ Thesis, INPG, Grenoble (2007).

14. R. Brooks, A. Corey, Hydraulic Properties of Porous Media, Colorado State University (1964).

15. A. W. Bishop, G. E. Blight, Some aspects of effective stress in saturated and partly saturated soils. Géotechnique, 13, 177-197 (1963).

16. M. Morvan, H. Wong, D. Branque, An unsaturated soil model with minimal number of parameters based on bounding surface plasticity. international journal for numerical and analytical methods in geomechanics, 34, 1512-1537 (2009). 